\title{
Anticipation in the Structure of the Solving Problems Skill
}

\author{
Assoc. Prof. Veselina Ivanova PhD
}

Assist. Prof. Maria Temnikova PhD

Trakia University, Faculty of Education, Bulgaria

Doi: 10.19044/esj.2018.c5p2

URL:http://dx.doi.org/10.19044/esj.2018.c5p2

\begin{abstract}
In the study the accent is put on the significant role of the foreseeing in the heuristic aspects of the solving problems activity. Theoretical formulations are deduced and the possibility of its purposeful formation is well-founded on the basis of the developing training particularities by the solving problems process.

In order to attain the goal the following methods are used: content analysis, pedagogical observation, pedagogical experiment, method of the determination of a success standard and a correlation degree.

The obtained results of the theoretical and experimental work are directed to the integration with the intellectual development of children and pupils.
\end{abstract}

Keywords: Anticipation, skills, scholar problems solving, developing training

\section{Introduction}

Essential characteristics of the stages of the process of solving problems skill formation

The infinity and the variety of the purposes and situations determining the different skills specificity predetermine also the difficulty in finding the universal conditions, objective laws and approaches for their formation. But the examination of the conceptions of their step-by-step development shows a close similarity. In his dissertation study P. Petrov relies on the conceptions of Hubert and Stuart Dreyfus as well as on those of Boris Minchev (Minchev, 1991) and describes the following stages determining some of their specific characteristics:

In the first stage "the beginner" realizes the purpose. Although the active examination of the problem object field its different components are perceived separately but with a wrong idea of entireness. An algorithm of the 
executed actions is given or the trainee finds it himself. His capacity to listen to and to ask questions is limited.

By the increase of the experience of coping with the situation /problem solving/ in the second stage "the advanced beginner" finds or he is given the important components. He observes the rules during the most part of the execution. The trainee does not associate the accumulated at that stage multitude of situation aspects with precise and universal rules. The heuristic operational structures of the solving problems skill are "related" to concrete problems.

In the third stage called "competence" the executor learns or is learned to make a choice and to take decisions. The beginning of the capacity to remember whole situations /problems-components/ is put. The situation understanding is not yet related to effective actions on a behaviour level. The particularities of the solving problems skill on an active plane are slightly verbalized.

In the fourth stage called "experience" the situation is perceived as similar to the already known one. The trainee sees what is necessary to be done and he has to take decisions concerning the execution way. He has already a combination of approaches for a various effect. At that stage criteria about the interchangeability of the execution quantity and quality arise. In the solving problems skill a great variety of algorithms and methods are worked. Some heuristic approaches are verbalized and/or mastered at a stage of arbitrariness.

In the fifth stage called "expertise" the expert understands, acts and learns spontaneously through the results by powerful heuristic processes without realizing that process. In the solving problems skill all that is fulfilled by habits, visual ideas and short mental chains. At that stage one goes from a direct control of the situation to inner forms of control which leads to the skill bahaviour components transfer. The control when solving problems is spread from the result to the initial solving stages. The situation understanding by the expert begins to divide a class of situations into subclasses each one of them sharing the same purpose and often the same solving method, a heuristic approach. A process of revealing of situation similarities begins which have seemed up to now incompatible. "The image of problem world" is created completely ( Petrov, 2013).

The practice shows that the duration of the formation of the solving problems skill, the limited scholar time and other factors permit the trainee to achieve the third stage. These objective laws require decisions in the didactic structure according to the different methodologies of the educational fields.

The most problematic part of each theory of the study stages is the transition between them. Scientists working on these problems reckon that the transitions may be facilitated using strategies, approaches and 
technologies especially if all this happens in compliance with the forthcoming stage (Vitanov, 1999). The following approaches are known: constructive approach, personally orientated approach, interactive methods application, study productive strategies etc. On the plane of the principal determinant - the purposes, a transition from the academic abstract knowledge to more practically orientated knowledge, skills and competencies and a training model focused on the study with understanding is obtained.

According to V. Petrova "... the constructive study involves in an active execution of various cognitive and practical actions. The creation of his own idea and the sense deriving from the acquired knowledge are obtained by forms and methods stimulating predominantly the understanding and the creation of his own logical construct before the memorizing and the reproduction" (Petrova, 2005).

I.

Poyia (1972)/ describes most often the "macromodel" of the process of "solving" with the following clearly distinguishable and specific moments:

- $\quad$ problem understanding;

- $\quad$ idea arising and plan creation /a decision search/;

- plan realization;

- $\quad$ additional work on the problem after the answer obtaining.

In this order the mental processes characterizing these stages are the following: understanding, foreseeing when searching for the decision, correct conclusions building, reflection on the problem and its solving. According to some authors in terms of practice the processes of foresight/prefeeling and prognostication/ (Petrov, 2011) and the reflection are slightly studied (Zhelev, Petrov, 2010).

Especially interesting for us is the study of the anticipation /foresight/ manifestations as a main sign of the skill during the solving problems process and the search for mechanisms of its formation and development directed to the integration with the intellectual development of primary school children and pupils.

Our motive for this focus is the view on the skills on an active and operational plane. Minchev (1991) distinguishes three groups of components in the skills structure: /1/ sense of situation, /2/ metacognitive actions and /3/ simple and complex habits of perceptive, memory and motor nature. The metacognitive components /one of which is the anticipation/ are considered to be those "stimulators" of the analytical and combinatory activity which participate in the formation of subpurposes and initiate the will efforts for the 
realization of transforming actions new for the subject experience (Ivanova, 2016).

\section{Foreseeing as a main sign of the solving problems skill}

Anticipation /prefeeling, foreseeing, presentiment, foresight, supposition for actions, events, results of experience, study, preliminary idea or a general notion of them/ is described as an "appearance in the mind before perceiving the separate concrete things; forestalling when by a mental motion a defined action may be executed before the appearance of the expected signal of it (Desev, 2003).

Lomov relates the anticipation to the capacity of taking different decisions and acting in time and space by "foreseeing" an expected future event. I.e. anticipation may be considered as a sign of each activity form - as a foresight of the final purpose or subpurposes, of the action alternatives and stages, as a preliminary rationalization of the action consequences or of the activity effects by making a critical evaluation of the past experience

The following levels of anticipation are known: subsensory, sensomotor, perceptive, conceptual /by generalizations, abstractions, calculating operations, acquired experience/ and verbal-mental one which is considered to be the highest level.

According to Zeltz each problem creates some idea of the purpose containing the decision in the form of an "urging blank". During an experiment of Luria the foreseeing is examined on the sensomotor plane in its quality of interpolating /complementing/ mechanisms which are a product of the structures transformation process similar to the already known ones by the subject (Lalov, 2003). During that experiment the significant role of the foreseeing in the heuristic aspects of the solving problems activity and the possibility of its purposeful formation is well visible. There is an analogical experiment through problems referring to the mounting and dismounting of parts (Piryov, 1969).

Key words and phrases of the description: manipulation, sight and foresight, generalization of the foreseeing mechanisms, spontaneity and entirety.

The given examples show the important role of the foreseeing when solving problems. Some authors add also the psychic models capacities functioning on the basis of the foreseeing mechanisms.

The development of the cybernetic ideas and methods when the main question is about the regulation and the self-regulation permits to see "insight" - deeply in the understanding of the foreseeing regulation functions as a sign of the capacity of solving problems and an element of its operational structure. The motives for that thesis are given by the 
fundamental idea of Lomov and Surkov (1980) according to whom the hypothesis use efficiency when solving problems depends on those conditions which determine the balance between the prognostication processes and the control operations. Petrov (2013) defines as "prognostication" the foreseeing part which to a great extent is realized and verbalized. When solving problems the prognostication is examined as a process of anticipating information receiving in the form of probable deductions about the object based on scientifically motivated situations and methods.

60 students of the Faculty of pedagogy of the Trakia University, Stara Zagora, Bulgaria have participated in the study. They study in the specialties of Preschool and primary school pedagogy and Primary school pedagogy with a foreign language - Bachelor educational and qualification degree. In the methodological system of work applied in the scholar subject of Training in thinking by mathematical problems solving are included a series of problems. By their solving different elements of the students' mathematical competency are built. Those problems are mutually complementing and create a "complex repeating situation" (Petrov, Temnikova, 2016). Examples of the different kinds of problems and some of their particularities are given below.

First kind of problems: In a given class there are 25 pupils. 14 of them play football, 15 practice swimming and 9 practice both sports. How many pupils practice neither football nor swimming?

At the first stage during the solving process the students understand the problem content and build a general hypothesis. At the second stage they generate a lot of ideas. In this concrete case - two ideas using the modeling method. During the first idea the line of natural numbers is used on which are consecutively marked the first 14 pupils who play football. The last 9 of them are marked as practicing both sports. On the axis are marked also the remaining 6 ones who practice swimming. That does not impede the reasoning because a number which does not depend on the objects layout is searched for. The number of the pupils who practice neither football nor swimming should be determined. During the second idea of solving the theoretical multiple approach and the diagrams of Oyler-Ven for the situation modeling presented in the problem are used. Both ways use heuristic approaches and modeling for the temporary situation simplification and the creation of conditions of making a problem solving plan.

When solving the second kind of problems the analytical approach /solving from the end/ is used. For example: From a plate with apples Anne has taken the half and one apple more. Then Pam has taken the half of the remaining apples and 2 apples more. In the end George has taken the half of the remaining apples and the last 3 apples. How many apples there were at 
the beginning and how many apples has taken each of them? A number of ideas for the problem solving are related to the following important moments: to begin the solving from the end; to introduce suitably the variables and to solve the problem by an equation; the idea of substitution may be illustrated; the searching characteristics may be prognosticated and the decision may be found.

When solving the third kind of problems included in the series the method of the invariant is used. One of the problems is the following: Two baskets contain 8 peaches each one. Nora has taken from the one a couple of peaches. Lili has taken from the other as many apples as the remaining quantity in the first basket. How many apples totally have remained in both baskets?

When solving the problem two trials are done on the basis of which the following supposition is formulated: no matter how many apples has taken Nora in the beginning 8 apples remain totally. The supposition is supported examining one case more and thus it is well-founded to a greater extent. When describing the decision finding the following terms are used: supposition, reasons for the supposition formulation, additional argument, thinking in the form of "if..., so". The decision may be presented in a table where all cases are examined, i.e. different invariants. The situation simplification in this problem is made by its visual presentation combined with the algebraic expressions modeling.

The following general conclusion concerning these problems may be made:

- $\quad$ the foreseeing as a main characteristic of the solving problems skill expressing its active nature is manifested mainly in two modi: sensomotor modus and a prognostication by solving methods and ideas;

the approach prognostication /multiple ideas/ is the main moment in the heuristic searching for strategies.

The observation made by us of the process of the decision searching for shows the particularities of its different stages and reveals some possibilities of purposeful formation of the trainees' anticipation capacities.

\section{I stage. Problem understanding and a general hypothesis building}

The problem solving begins by its understanding. As a result of the analysis of the condition and the searching for in the problem a general hypothesis arises in its quality of an initial idea. In the content aspect it includes a prognostication of the relations between the groups of objects under the given conditions and some general ideas of the solving way. The general hypothesis determines the searching field and becomes the initial point of the second stage. 


\section{II stage. General hypothesis development and formation of a multitude of solving ideas}

The heuristic approaches are applied partially at the first stage but mainly at the second one, such as: temporary situation simplification, problem "specialization' by examining the different private cases, reformulation of the search in the problem, solving "from the end" and various analogies use. The cognitive activity is regulated by them but the observations show that this is to a certain extent. The operations become less chaotic.

\section{III stage. Obtaining the main /specified/ hypothesis}

At this stage an evaluation of the multitude of solving ideas is made and the most perspective one is chosen. It becomes detailed on the basis of the characteristics of the objects in the different groups. A decision projection follows.

The experimental work done shows the following: if one admits that a comparison regulatory mechanism functions, about its two phases /hypotheses building and their resulting evaluation/ may be said that they are characteristic of each stage of the search. The following conclusion may be made: the prognostication by hypotheses building is the main part of the selfregulation at each stage of the search.

The different kinds of heuristic procedures create conditions for the development of the comparison of the hypotheses with the results of the operations especially at the initial search stages when the information is insufficient.

It is known that the two main heuristic characteristics are "directing" to the solving idea and "shortening" of the choice of the different possible ideas. The direction to the solving idea is observed at the first and the second stages /when formulating likely hypotheses/. The search shortening is realized by the creation of likely hypotheses, through their confirmation and by the elimination of ideas from the multitude. These actions lead to the two following conclusions:

- $\quad$ a relation between the solving efficiency and the number of ideas in the multitude of alternatives can not be searched;

- $\quad$ the solving idea may be the only one but with a higher degree of likeliness and it may lead to the effective decision. 
For that model of the decision search activity it is characteristic that the main actions included in it give possibilities of realizing the prognosticating, directing and shortening heuristic functions.

\section{Conclusion}

Theoretical formulations and some practical aspects of the anticipation problem on the basis of the developing training particularities through the solving problems process are derived and systematized.

By the created methodological work system combining productive and reproductive strategies, approaches, methods and means the student is put in the center of an active cognitive process during which he constructs his ideas for mathematical problems solving on the basis of his available knowledge and past experience.

By its application during the lessons of Training in thinking by solving problems the students achieve higher levels of the inner purposeful orientation providing efficiency when forming the anticipation capacities having the trend toward a transfer in the ages and the activities.

The students' anticipation development contributes to the following: creation of their transversal competencies and realization of the knowledge and skills transfer by solving a limited number of problems in close fields, formation of the style of work generating the inner motivation for the non standard mathematical problems solving.

\section{References:}

1. Vitanov, L. (1999). Productive strategies of the engineering and technology training in the initial classes of the secondary school. Sofia: Veda-Slovena - ZhG.

2. Desev, L. (2003). Dictionary of Psychology. (p.48). Sofia: Bulgarica.

3. Dreyfus, H.I., S.E. Dreifus. (1986). Putting computers in their place. (p. 53). Social research.

4. Ivanova, V. (2016). Metacognitive components in the structure of the abilities of motor education. Proceedings of the III International scientific and practical conference "Topical problems of modern science and possible solutions /September 28 - 29, 2016, Dubai, UAE/", No 10/14/, vol.5, October 2016, (p. 8-10). htpp://wsconference.com/.

5. Kuljutkin, Ju. (1970). Heuristic methods in the solving structure. Pedagogika: Moskva.

6. Lalov, B. (2003). Extrapolating theory of training. (p. 11). Sofia.

7. Lomov, B., \& Surkov, E. (1980). Anticipation in the activity structure. (p. 202). Moskva: Nauka.

8. Luriya A. Language and consciousness. (1979). (p. 320). 
9. Minchev, B. (1991). Situations and skills. (p. 176). Sofia: Universiry press St. Kliment Ohridski.

10. Petrova, V. (2005). Children's concepts during the training of natal country, environment and human being and nature in the $1^{\text {st }}-4^{\text {th }}$ class. (p. 37). Stara Zagora: Kota.

11. Petrov, P. (1996). Didactic aspects of prognosing during the search for resolutions of mathematical tasks. Ownstudy of a dissertation for the acquiring of a PhD. Stara Zagora: Trakia University, Faculty of Education.

12. Petrov, P. (2013). Didactical dimensions of the tasks solving skill. Ph.D. study, Stara Zagora: Trakia University, Faculty of Education.

13. Petrov, P. (2011). Methodology of the mathematical training in the primary classes /reflection of the concept of the problems solving skill/. (pp. 159-166). Stara Zagora: University press.

14. Poyia D. (1972). How to solve a problem?. Sofia: Narodna prosveta.

15. Piryov, G. (1969). Understanding degrees when solving technical problems. (pp. 224-230). Sofia: Narodna prosveta. 